\title{
Thin-Layer Matrix Sublimation with Vapor- Sorption Induced Co-Crystallization for Sensitive and Reproducible SAMDI-TOF MS Analysis of Protein Biosensors
}

\author{
Michael J. Roth, Jaekuk Kim, Erica M. Maresh, Daniel A. Plymire, John R. Corbett, \\ Junmei Zhang, Steven M. Patrie
}

Department of Pathology, University of Texas Southwestern Medical Center, Dallas, TX 75390, USA

\begin{abstract}
Coupling immunoassays on self-assembled monolayers (SAMs) to matrix-assisted laser desorption/ionization time-of-flight (MALDI-TOF) mass spectrometry (MS) provides improved assay selectivity compared with traditional photometric detection techniques. We show that thinlayer-transfer (TLT) of a-cyano-4-hydroxycinnaminic acid (CHCA) MALDI matrix via vacuum sublimation followed by organic solvent-based vapor-sorption induced co-crystallization (VIC) results in unique matrix/analyte co-crystallization tendencies that optimizes assay reproducibility and sensitivity. Unique matrix crystal morphologies resulted from VIC solvent vapors, indicating nucleation and crystal growth characteristics depend upon VIC parameters. We observed that CHCA microcrystals generated by methanol VIC resulted in $>10 \times$ better sensitivity, increased analyte charging, and improved precision compared with dried droplet measurements. The uniformity of matrix/analyte co-crystallization across planar immunoassays directed at intact proteins yielded low spectral variation for single shot replicates (18.5\% relative standard deviation, RSD) and signal averaged spectra ( $<10 \% \mathrm{RSD})$. We envision that TLT and VIC for MALDI-TOF will enable high-throughput, reproducible array-based immunoassays for protein molecular diagnostic assays in diverse biochemical and clinical applications.
\end{abstract}

Key words: MALDI, SAMDI, Protein analysis, Reproducibility

\section{Introduction}

Tmmunoassays are used in a variety of biochemical and clinical applications, including protein expression assays, biomarker validation analyses, and enzyme kinetics [1-3]. Detection methods include colorimetry, radioactivity, fluo-

Electronic supplementary material The online version of this article (doi:10.1007/s13361-012-0442-7) contains supplementary material, which is available to authorized users.

Correspondence to: Steven Patrie; e-mail: steven.patrie@utsouthwestern.edu rescence, chemiluminescence, electrochemical, quartz crystal microbalance (QCM), and surface plasmon resonance (SPR) [4-6]. These methods are sensitive and scalable, often enabling quantitative measurements in parallel and with excellent reproducibility. However, since assay selectivity is dependent upon an antibody, these methods are susceptible to interferences that can lead to false positive or negative readings.

Immunoassays adapted to MS benefit from the enhanced selectivity provided by molecular mass determination, improving specificity and enabling unintended, unknown, or multiplexed components to be identified [7, 8]. Nelson and coworkers have pioneered MALDI-TOF immunoassays using antibody-bound microcolumns to isolate antigens from 
samples followed by elution to a MALDI plate [9]. Others have performed MALDI-TOF directly on antigen-bound media (e.g., surface-enhanced laser desorption ionization (SELDI-TOF) MS and immuno-MALDI (iMALDI)) [10, 11]. The work highlights that immunoassays with MALDITOF necessitate specialized solid-phase substrates (e.g., porous frits, affinity beads, planar surfaces) and chemistries (e.g., self-assembled monolayers (SAMs) [12], nitrocellulose films [13]) to optimize speed and reliability of target enrichment and MS detection sensitivity.

While MALDI-immunoassays demonstrate robust detection of target antigens, the methods typically suffer from poor quantitative reproducibility using conventional dried droplet (DD) matrix deposition. DD leads to non-uniform analyte/matrix co-crystallization forming "sweet spots" which reduce inter- and intra-assay reproducibility [14-16]. Alternative matrix deposition methods have been reported to enhance homogeneity of matrix/analyte co-crystals [17-28], although MALDI-TOF measurements typically yield precisions above $20 \%$ relative standard deviation (RSD). Performing relative quantitation against an internal standard at similar molecular mass to the target analyte improves MALDI precision to $<10 \% \operatorname{RSD}[29,30]$.

Monolayers provide a unique platform for diverse biochemical assays, support multiplexed processing via high-density arrays, and are amenable to MALDI-TOF MS (denoted SAMDI-TOF MS) [31-33]. Previous SAMDI-TOF immunoassays incorporated SAMs composed of alkanethiolates on gold functionalized with polyethylene glycol groups and metal binding functional groups to facilitate antibody immobilization [33]. These previous immunosensors were prepared by immobilizing his-tagged protein $\mathrm{G}$ onto $\mathrm{Ni}^{2+}$ bound SAMs, followed by binding of IgG antibodies targeted to the analyte of interest. The work demonstrated analyses of a variety of intact proteins, protein complexes, and PTMs in biofluids, with minimal non-specific binding owing to the surface chemistries used [33]. Compared with traditional adsorption methods that randomly coat antibodies onto substrates, SAM chemistries can be tailored for improved control of antibody orientation and surface density to maximize fractional activity of antibody-antigen interactions and enhance loading capacity [7]. Such precise control over surface characteristics enables reproducible, quantitative measurements with SPR or QCM immunoassays; however, internal standards were still necessary to improve reliability of MALDI measurements [34].

This work focuses on reducing MALDI-TOF spectral variability for protein immunoassays without the use of internal standards (i.e., "label-free" processing). Recent work suggests that MALDI crystals with a narrow size distribution yield improved reproducibility, resolution, sensitivity and signal-to-noise ratio $(\mathrm{S} / \mathrm{N})[35,36]$. Based on this work, we postulated that controlled distribution of immobilized proteins on SAMs with uniform MALDI matrix crystallization would improve SAMDI-TOF spectral reproducibility and sensitivity. We adapted common strategies used in materials science to control MALDI matrix deposition and matrix/analyte co-crystallization. Here we show that "thin-layer transfer" (TLT) of matrix via vacuum matrix sublimation combined with organic solvent-based "vapor-sorption induced co-crystallization" (VIC) enables generation of homogeneous matrix/analyte co-crystals, resulting in improved sensitivity and reproducibility compared with DD matrix deposition.

\section{Experimental}

\section{Materials and Reagents}

Detergents, MALDI matrix, and protein standards were purchased from Sigma-Aldrich (St. Louis, MO, USA). All organic solvents used were of HPLC grade, and ethyl alcohol to store and wash SAM plates was absolute anhydrous 200 proof. Water was purified to a resistance of $18 \mathrm{M} \Omega$. The $6 \times$ His-tagged protein $\mathrm{G}$ was purchased from Biovision Inc. (Mountain View, CA, USA). IgG antibodies to human serum albumin, cystatin C, myoglobin, and transferrin were purchased from Bethyl Laboratories Inc. (Montgomery, TX, USA). Human pooled normal plasma was purchased from Precision BioLogic (Dartmouth, Nova Scotia, Canada). HEPES buffer was purchased from Cellgro (Herndon, VA, USA) and phosphate buffered saline (PBS) was purchased from Gibco (Grand Island, NY, USA). Microscope cover glasses $(24 \times 60 \times 0.15 \mathrm{~mm})$ were purchased from VWR Scientific Inc. (Media, PA, USA).

\section{Immunoassay Procedure}

Nitrilotriacetic acid (NTA) SAMs were generally prepared as described previously [33]. Briefly, $24 \times 60 \mathrm{~mm}$ microscope cover glasses were cleaned with a residue-free, phosphate-free detergent (Fisher Scientific, Pittsburgh, PA, USA) prior to gold coating. Glass slides were metalized with $30 \AA$ of chromium followed by $300 \AA$ of gold using a CHA Mark 50 E-gun evaporator. Monolayers were generated at room temperature overnight by immersion of gold slides in an ethanolic solution containing triethylene glycol (EG3)$\mathrm{OH}$ and EG3-NTA terminated alkanethiolates. Surface density of NTA was estimated by the EG3-OH to EG3NTA solution ratio. The SAM-covered gold slides were cut into rectangular pieces measuring $4 \times 5 \mathrm{~mm}$. Immunoassays were generated by treating the surface with $10 \mathrm{mM} \mathrm{NiSO}_{4}$ $\left(\mathrm{H}_{2} \mathrm{O}, 0.01 \%\right.$ Tween-20) for $30 \mathrm{~min}$ to generate NTA/ $\mathrm{Ni}^{2+}$ complex, followed by immobilization of $0.77 \mu \mathrm{M}$ Histagged recombinant protein $\mathrm{G}$ (PBS, pH 7.2, $0.01 \%$ Tween) for $30 \mathrm{~min}$ and then $0.33 \mu \mathrm{M} \mathrm{IgG}$ antibody (PBS, $\mathrm{pH} 7.2$, $0.01 \%$ Tween-20) for 30 min. Plasma was diluted with PBS or HEPES buffer with $0.01 \%$ Tween-20 and incubated at room temperature for 1-3 h. All incubation steps (except SAM generation) were performed by spotting solution onto the surface. Chips were rinsed with a stream of $18 \mathrm{M} \Omega$ water 
after each incubation step to remove residual solution and decrease non-specific interactions, followed by drying with a gentle stream of $\mathrm{N}_{2}$ gas. For DD matrix deposition, a thin layer of CHCA solution in the solvents described in the results $(0.3 \mu \mathrm{L}, 10 \mathrm{mg} / \mathrm{mL}$ in organic solvent) was applied to each chip and dried prior to MALDI mass analysis.

\section{Thin Layer Transfer of MALDI Matrix}

A custom device was used for thin-layer transfer (TLT) of matrix via vacuum sublimation. A hotplate (VWR International, West Chester, PA, USA) was placed inside of a transparent vacuum chamber (Thermo Fisher Scientific, Rochester, NY, USA). A $2 \mathrm{mg} / \mathrm{mL}$ solution containing MALDI matrix in acetone was uniformly spread on a "TLT" glass slide $(47 \times 57 \times 0.2 \mathrm{~mm})$ using a pipette. The use of CHCA as a matrix for sublimation is common due to the good thermal properties [37] and mass spectral performance, although other matrix molecules may also be deposited by sublimation [23, 28]. The TLT slide with matrix was placed on top of the hotplate. SAM biochips were attached to an aluminum cooling mantle $(170 \times$ $115 \times 33 \mathrm{~mm}$ ) positioned $1 \mathrm{~cm}$ away from the TLT slide. The cooling mantel was maintained at $10{ }^{\circ} \mathrm{C}$ by a recirculating water cooler (Thermo Fisher Scientific, Rochester, NY, USA). The vacuum chamber was evacuated to $\sim 125$ Torr and the hotplate was gradually heated to allow the matrix on the TLT slide to sublime. Typically, matrix sublimation initialized at $\sim 140{ }^{\circ} \mathrm{C}$ and completed at $\sim 160{ }^{\circ} \mathrm{C}$ (total TLT time: $\sim 3.5 \mathrm{~min}$ ). Under these conditions, matrix transferred uniformly to the SAM biochip with minimal spatial dispersion. The surface density of sublimed matrix was controlled by the amount of matrix deposited onto the TLT glass slide. Upon completion of TLT the hotplate was turned off, vacuum pressure was released, and the SAM biochips were removed and allowed to cool to RT within $1 \mathrm{~min}$.

\section{Vapor-Sorption Induced Co-Crystallization}

Biochips with CHCA deposited by TLT were treated with a variety of solvent vapors including water, methanol, ethanol, acetone, acetonitrile, isopropanol, dimethyl sulfoxide (DMSO), and $N, N$-dimethylformamide (DMF). Solvent properties relevant to the VIC process are summarized in Supplementary Table S1. To achieve matrix/analyte cocrystallization, a solvent chamber $(80 \mathrm{~mm}$ o.d. $\times 40 \mathrm{~mm})$ was filled with $30 \mathrm{~mL}$ solvent. The biochips were placed at the top of the solvent chamber and exposed to vapors at controlled temperatures and time (see Supplementary Table S2). The VIC completion time was empirically determined and coincided with an optical transition (determined visually) of the sublimed amorphous matrix to a glass state. Upon completion of the VIC process the SAM biochips were allowed to air dry at RT to enable matrix/analyte cocrystallization. The typical drying times were $<1 \mathrm{~s}$ for all solvents except DMSO ( $\sim 3 \mathrm{~min})$ and DMF $(\sim 5 \mathrm{~s})$. After drying, the SAM biochips were attached to a membrane sample plate, \#V700698 (AB SCIEX, Foster City, CA, USA) with vacuum grease for MALDI-TOF analysis.

\section{Mass Spectrometry}

All mass spectra in this study were obtained using a Voyager DE-PRO MALDI-TOF mass spectrometer (Applied Biosystems, Framingham, MA, USA) in linear mode. The instrumental parameters were as follows: accelerating voltage $(\mathrm{AV}): 25 \mathrm{kV}$, grid voltage: $93 \% \mathrm{AV}$, guide wire: $0.15 \%$, and extraction delay time: 510 ns. A $337 \mathrm{~nm}$ nitrogen UV laser (pulse width: $3 \mathrm{~ns}$, pulse energy: $120 \mu \mathrm{J}$ $\max$ at $10 \mathrm{~Hz}$ ) was used at a repetition rate of $3 \mathrm{~Hz}$ for sample ionization. The laser energy was tuned for each matrix deposition method. The laser irradiation area was estimated to be $\sim 80-100 \mu \mathrm{m}^{2}$ with a substrate incidence angle of $\sim 30^{\circ}$. Mass spectra were generated from 100 laser shots and were stored as raw data without further manipulation unless otherwise stated. To generate calibration curves for albumin and spiked myoglobin, advanced baseline correction in the Data Explorer software (ver. 4.9) was used for peak height measurement. Mass spectra were externally calibrated with equine myoglobin, bovine ubiquitin, and bovine serum albumin. For all deconvoluted plots, data points represent the average of three spectra, unless otherwise stated.

\section{Light Microscopy, SEM, and AFM}

The matrix crystal morphologies of the TLT/VIC surfaces were measured by light microscopy, scanning electron microscopy (SEM), and atomic force microscopy (AFM) to determine matrix thickness, crystal size, and shape. A Nikon MM-40 microscope (Nikon Instruments Inc., Melville, NY, USA) with a Sony CCD-IRIS color video camera (Sony Electronics Inc., New York, NY, USA) was used with $5 \times, 10 \times, 20 \times, 50 \times$, and $150 \times$ objective lenses. For SEM, MS immunoassays with sublimed matrix were pre-coated with approximately $10 \mathrm{~nm}$ of gold for $60 \mathrm{~s}$ in a sputter coater (Cressington 108auto, Watford, Hertfordshire, UK) at an argon gas pressure of 0.2 Torr and an applied current of $20 \mathrm{~mA}$. The matrix crystals were investigated at $10-15 \mathrm{kV}$ with an FEI XL30 environmental SEM (Hillsboro, OR, USA). For AFM, a VEECO Dimension 3100 atomic force microscope (Plainview, NY, USA) was used to characterize matrix film thicknesses.

\section{Results and Discussion}

\section{Thin Layer Transfer (TLT)}

Matrix deposition by sublimation has proven reliable for a variety of applications in the MALDI imaging field $[19,22,23,28,38,39]$. Conventional sublimation apparatuses for bulk chemical purification provide limit- 
ed control over thickness and in-plane uniformity of the matrix deposited. To overcome this, we developed a sublimation strategy that controls the matrix surface density on immunoassays with common laboratory equipment. The approach was designated as "thin layer (matrix) transfer" (TLT) to differentiate this specialized application from traditional bulk sublimation deposition; with TLT providing matrix surface densities of tens of $\mathrm{ng} / \mathrm{mm}^{2}$ to maximize sensitivity for detection of proteins in a monolayer. Model immunoassays against plasma proteins were used to compare TLT and DD matrix deposition [33]. Unlike DD, where inhomogeneous matrix crystallization was observed (e.g., Figure S1b), deposition of CHCA by TLT provides uniform matrix over the SAM substrate (Figure S1d), although no MS peaks were observed (Figure S1c) unless the surface rested for extended periods in humid environments (Figure S1e). The results suggested that TLT renders CHCA on SAMs in a state that does not facilitate ultra violet (UV) laser energy absorption and/or co-crystallization with the immobilized analyte.

\section{Vapor-Sorption Induced Co-Crystallization (VIC)}

Crystallization and Crystal Morphologies Organic materials deposited by vacuum sublimation are typically amorphous or exist in polymorphic crystalline states that are less thermodynamically stable than large monodisperse crystals [40]. We hypothesized that vapors from organic solvents would promote the crystalline transformation similar to methods used in materials research $[40,41]$. To test this, five polar solvents with varying CHCA solubility were evaluated for suitability for VIC and downstream SAMDI MS (Table S1). Each solvent vapor system was empirically optimized for duration of vapor exposure and solvent temperature during VIC (Table S2) using distinct visual cues such as change of surface color and/or transparency.

Light microscopy and SEM on these surfaces demonstrated diverse CHCA crystal morphologies specific to each vapor (Figure 1). DMSO VIC consistently yielded a "film" with planar spherulitic crystalline patterns (10$30 \mu \mathrm{m})$. DMF VIC led to surfaces with discrete crystals $(\sim 5 \mu \mathrm{m})$ separated by less defined crystalline film. Methanol, ethanol, and acetone VIC produced discrete and/or aggregated microcrystals (100-400 nm) with discriminating morphological characteristics observed for each solvent (Figure S2).

MS Analysis The MALDI-TOF performance was evaluated for VIC crystal morphologies using anti-albumin immunoassays exposed to either $10^{5}$ or $10^{6}$ plasma dilutions. Comparison of the deconvoluted albumin ion signals (Figure 2a) from representative mass spectra for each solvent (Figure S3) shows that CHCA microcrystals from methanol, ethanol, and acetone VIC provided the strongest antigen signal at both dilutions. The TLT/VIC treatment with all solvent vapors yielded better signal when compared with DD with CHCA suspended in the same solvents. TLT matrix deposition with methanol VIC yielded $2.3 \times$ and $17 \times$ higher ion yield compared with any DD CHCA/solvent combination at the $10^{5}$ and $10^{6}$ plasma dilutions, respectively. Preliminary testing has shown detection of various proteins at picomolar concentrations (Figure S4).

Spectral Discriminations Analyte discrimination is well known in MALDI, with previous work showing that matrix solution conditions and crystal morphology influenced analyte incorporation into the matrix crystal $[42,43]$. MALDI imaging investigations have shown that hydration of tissue samples prior to matrix deposition improved analyte incorporation into crystals and that analyte signal from tissues may be further tuned by application of solvent vapor post-crystallization [28]. Similarly, with TLT/VIC, discriminating mass spectral features were noted for the different crystal morphologies. Protein G, antibody, or antigen MS signals varied between TLT/VIC preparations; DMSO, and methanol VIC represented the extremes (Figure $2 b$ and $c$ ). For
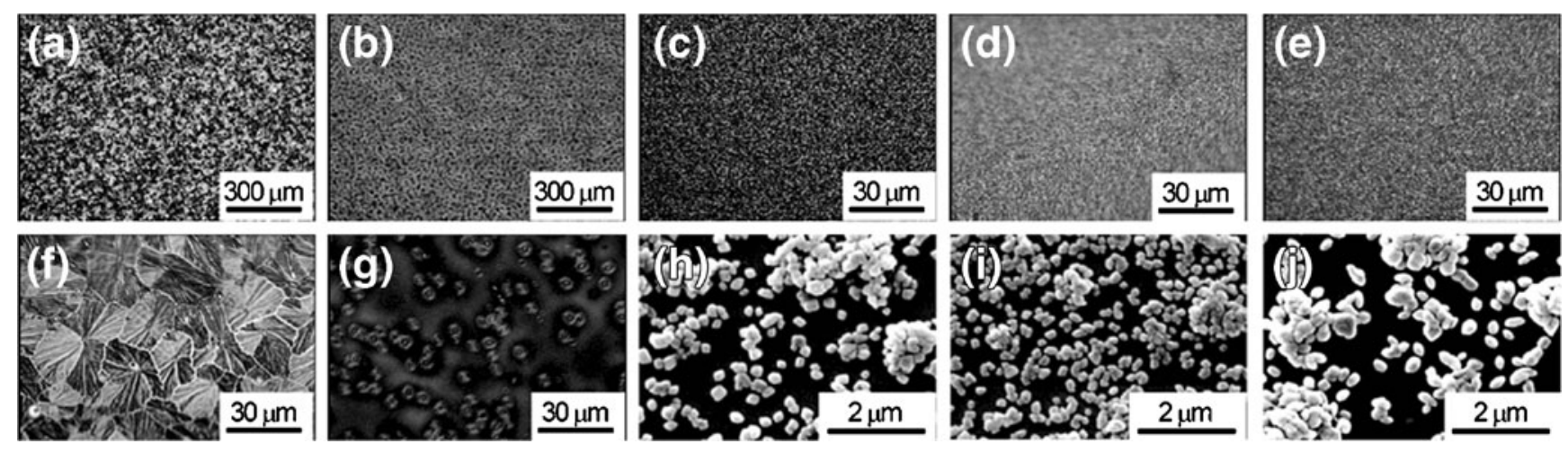

Figure 1. Light microscope and SEM images of CHCA TLT with various solvent VIC. (a) DMSO VIC, (b) DMF VIC, (c) methanol VIC, (d) ethanol VIC, (e) acetone VIC, (f) DMSO VIC, (g) DMF VIC, (h) methanol VIC, (i) ethanol VIC, (j) acetone VIC 
(a)

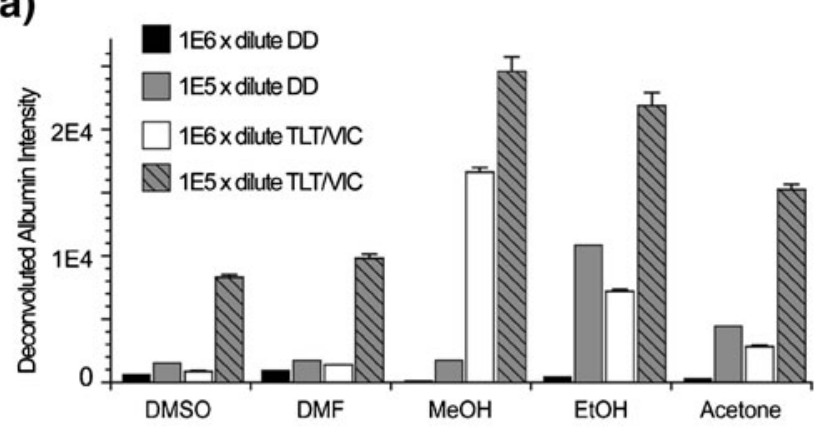

(b)

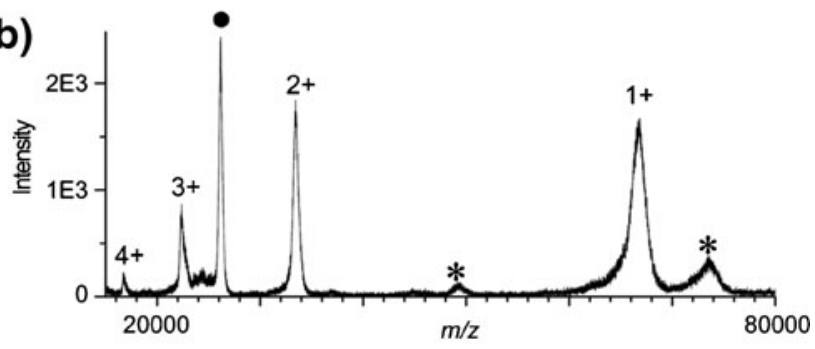

(c)

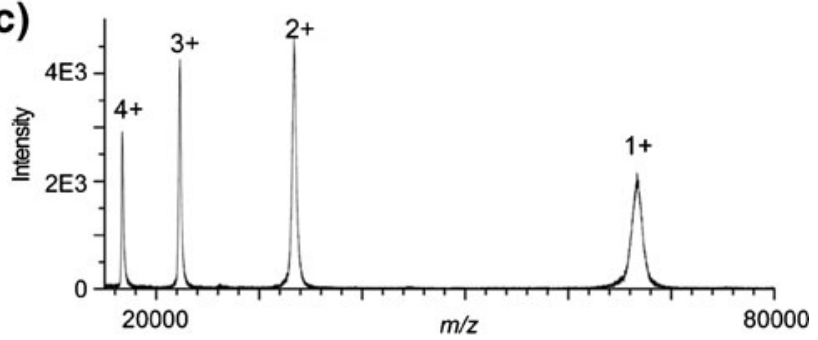

(d)

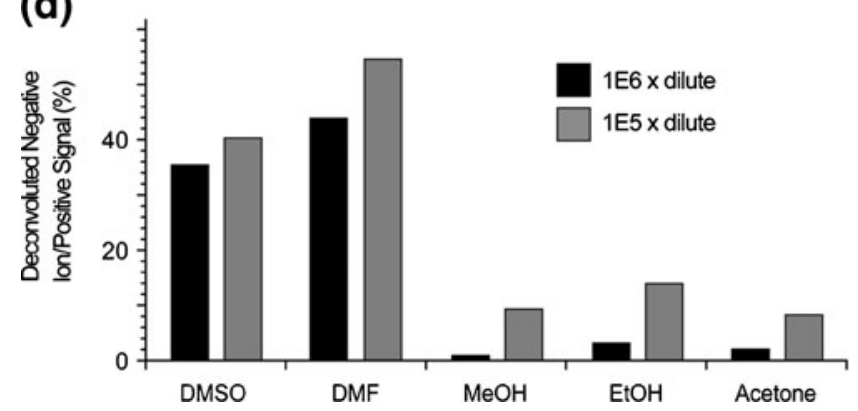

Figure 2. Comparison of albumin ion signal sensitivity for CHCA TLT followed by various organic solvent VIC. (a) Deconvoluted albumin ion signal intensity for $10^{5}$ and $10^{6}$ diluted plasma using DD and TLT/VIC with different solvents; error bars correspond to relative standard deviation (RSD). (b) Albumin spectrum generated from $10^{5}$ dilute plasma with TLT and DMSO VIC, and (c) $\mathrm{MeOH}$ VIC. (d) Negative ion mode deconvoluted albumin signal intensity using different VIC solvents. The ion peaks in (b) and (c) labeled with (•) and (*) are from protein $G$ and anti-albumin, respectively

methanol VIC, protonated ions derived mostly from the albumin while DMSO VIC produced spectra similar to the DD approach with protonated species of all surface proteins present. Similar results have been observed from other endogenous plasma protein immunoassays (unpublished).

The results also show the presence of multiply charged albumin ions differed for CHCA microcrystals versus films as described in Table S3. The data indicate that singly and doubly charged albumin species dominated spectra from surfaces with crystal films, while microcrystals provided spectra featuring albumin signals with higher charge. Decreased protonation from DMSO- and DMF-treated surfaces may have derived from charge neutralization by solvents with higher $\mathrm{pKa}$ or by increased competition between ablated surface components in the gas-phase plume [44]. Interestingly, similar experiments performed in the negative ion mode showed that decreased protonation for DMSO- and DMF-treated surfaces correlated with a higher proportion of negatively charged ions (Figure 2d). This suggests that protonated ion yields in CHCA films may have been reduced by the presence of photoelectrons or electrons that derived from metal surfaces [45].

Crystallization The results thus far provide insight into solvent dependent nucleation and crystal growth characteristics that affected endpoint CHCA products (e.g., microcrystals versus planar spherulites). Previous investigations have shown that crystalline transitions of vapor penetrated organic materials were highly dependent upon solubility, initial crystallinity of the material, and temperature [46]. Despite having low vapor pressures, the onset of VIC occurred by exposure to solvent vapor at room temperature for $60 \mathrm{~s}$ and $40 \mathrm{~s}$ for DMSO and DMF, respectively (Table S2), likely due to high CHCA solubility. At the onset of VIC the surfaces turned glassy and crystallization was completed by drying in the absence of the vapor at ambient temperature and pressure. CHCA crystal formation as a function of time using DMSO VIC was captured via light microscope (Figure S4). The results may indicate that DMSO vapor plasticized CHCA into a system that mimicked a supercooled liquid environment near its glass transition state [47]. Larger crystals separated by less defined films in DMF VIC (Figure 1) suggest that high heat loss at early nucleation sites promoted bulk matrix transport to the growing crystal where $3 \mathrm{D}$ crystal growth occurred by Ostwald ripening. The presence of all surface bound components in the mass spectra of DMF and DMSO treated surfaces suggests that vapor penetrants with high solubility easily diffused through the TLT layer to the surface to incorporate surface analytes into the growing crystals.

Methanol, ethanol, and acetone solvents have higher vapor pressures but lower CHCA solubility compared with DMSO and DMF. To increase the VIC transformation rate, these solvents required heating, presumably to better solvate the TLT surface. If complete dissolution of the CHCA particles from the TLT surface occurred in these solvents, then rapid drying $(<1 \quad \mathrm{~s})$ supports a high nucleation rate that limited the size of crystals. The selectivity of albumin in MS spectra with 
methanol VIC might suggest that complete dissolution of the surface proteins did not occur and the solvent served to change the molecular packing of already present crystals. In this case, the extent of analyte incorporation may be dependent on factors such as analyte/solvent compatibility and the location of the antigen near the matrix layer.

\section{Assay Optimization}

Matrix Surface Density Matrix surface density deposited by TLT provides an approximation of matrix/ analyte ratio $(\mathrm{M} / \mathrm{A})$, a parameter controlled in MALDI experiments. We sought to determine the influence of starting matrix surface density on crystal morphology and MS performance. A series of albumin immunoassays were treated with CHCA at matrix surface densities from 5 to $111 \mathrm{ng} / \mathrm{mm}^{2}$ followed by either methanol or DMSO VIC and MS analysis. A plot of the deconvoluted albumin signal versus matrix surface density (Figure 3a) shows maximum signal at $\sim 28 \mathrm{ng} / \mathrm{mm}^{2}$ for both solvents. SEM images for methanol VIC demonstrate a particle size distribution from $\sim 100$ to $300 \mathrm{~nm}$ at all surface densities (Figure 3b), with uniformly dispersed microcrystals up to $28 \mathrm{ng} / \mathrm{mm}^{2}$ and aggregation at higher surface matrix densities. With methanol VIC, the relatively sharp decrease in signal at higher matrix densities correlated with increased aggregation.

For DMSO VIC the signal decay beyond the optimal value linearly correlated with increased matrix density. Microscope images show that the spherulite planar dimensions were relatively consistent at all matrix surface densities (Figure 3c). Thickness measurements with AFM show that spherulites at $37 \mathrm{ng} / \mathrm{mm}^{2}$ were $\sim 28 \pm 3 \mathrm{~nm}$ (Figure $3 \mathrm{~d}$, left) in agreement with $26 \mathrm{~nm}$ thickness based on CHCA density calculations. The results indicate that TLT matrix deposition allowed quantitative transfer of matrix and M/A control in a manner that was consistent across the entire biochip surface.

Laser Irradiation Optimization Previous work suggests that the penetration depth of a UV laser is on the order of tens to hundreds of nm [48], similar to the size and thickness estimates for TLT/VIC generated crystals and films. Studies suggest that crystals of this size are completely volatilized by a single laser shot [35]. Here we examined how laser intensity affects antigen signal using immunoassays at $28 \mathrm{ng} / \mathrm{mm}^{2}$ CHCA surface density and crystallized with either methanol or DMSO VIC. Spectral waterfall plots (Figure 4) and the corresponding plot of deconvoluted albumin ion signals at different laser intensities (Figure 4b) show that past the threshold for ion formation, albumin signal rises to a maximum and then decays rapidly. The optimal signal occurred at slightly higher laser fluence for the spherulites than microcrystals, indicating that surfaces with microcrystals benefit from reflected light by exposed gold. With laser drilling experiments we noted strong

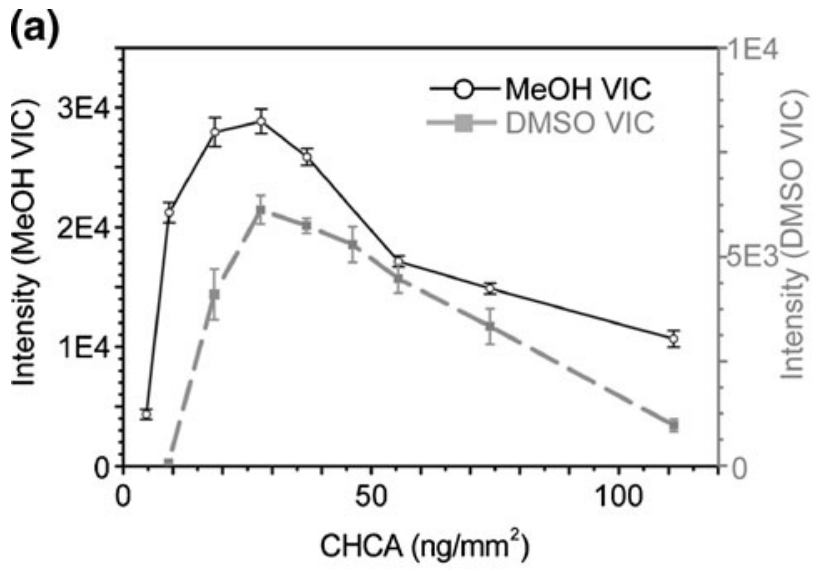

(b)

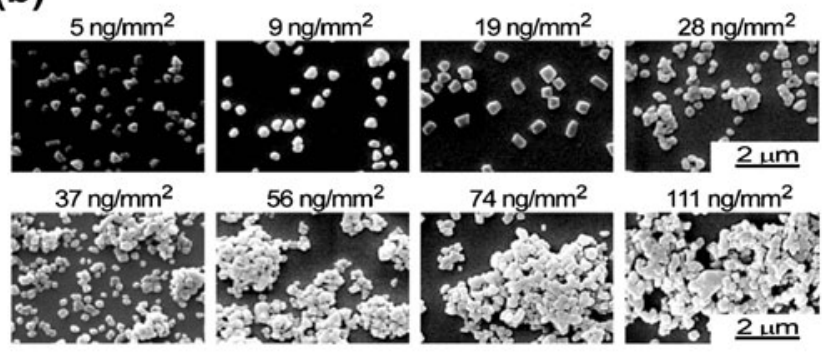

(c)

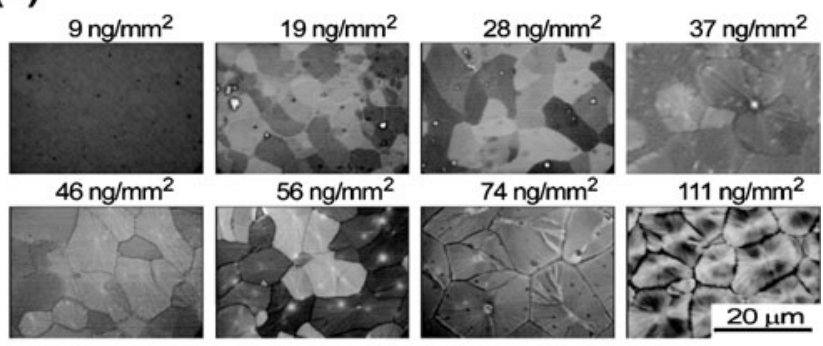

(d)
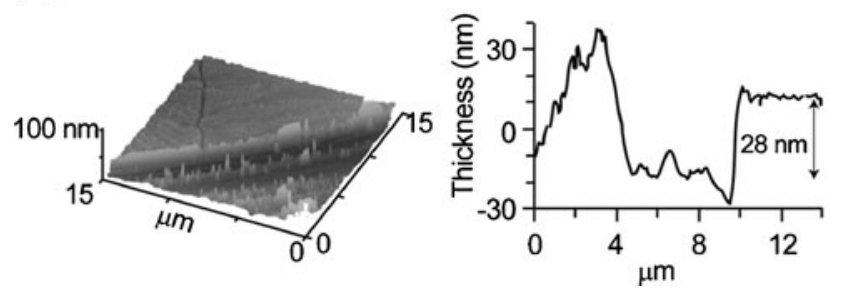

Figure 3. Characterization of signal sensitivity and crystal morphology as a function of CHCA TLT surface density. (a) Deconvoluted albumin signal for albumin in $10^{5}$ diluted plasma by $\mathrm{CHCA}$ sublimation followed by methanol (left axis) and DMSO (right axis) VIC. (b) SEM images of sublimed CHCA followed by methanol VIC. (c) Light microscope images of sublimed CHCA followed by DMSO VIC. (d) AFM images of $37 \mathrm{ng} / \mathrm{mm}^{2}$ CHCA TLT with DMSO VIC demonstrating $28 \mathrm{~nm}$ film thickness

association between analyte signal and successive laser shots (Figure S6). For surfaces at $28 \mathrm{ng} / \mathrm{mm}^{2}$ and $37 \mathrm{ng} /$ $\mathrm{mm}^{2}$ matrix density with methanol VIC, near maximal signal was observed for only the first two and three shots, 
respectively. Similar results were observed with the DMSO except the first shot appeared to peel the outer layers of matrix from the surface exposing the analyte near the surface. The laser fluence results contrast traditional MALDI assays where analyte signal tends to increase with laser fluence until detector saturation occurs [48]. The current data suggest that poor heat conduction in the small crystals causes excess energy deposition directly into the analyte, resulting in thermal decomposition and decreased analyte signal. The laser drilling experiments show that analyte was consumed quickly, indicating that to maximize $\mathrm{S} / \mathrm{N}$ in

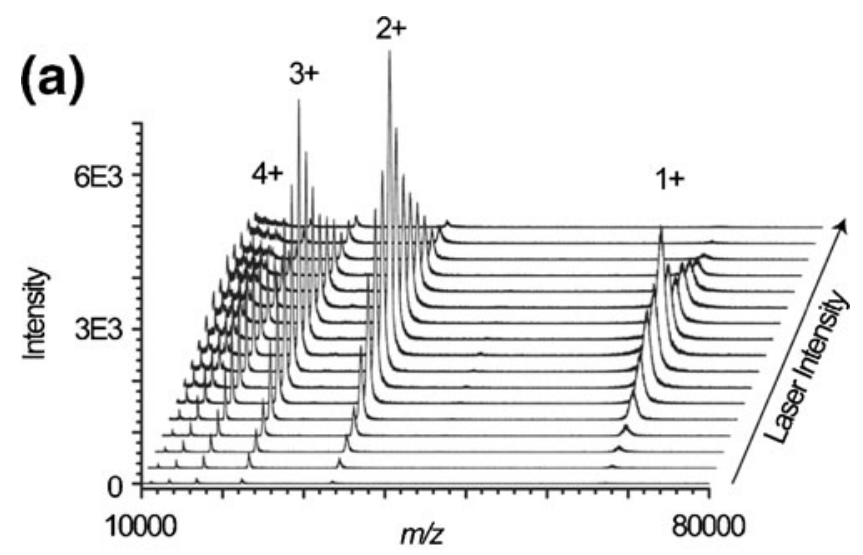

(b)

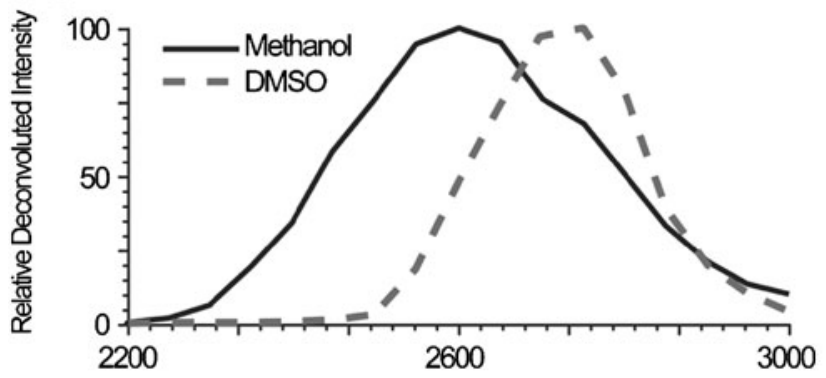

(c)

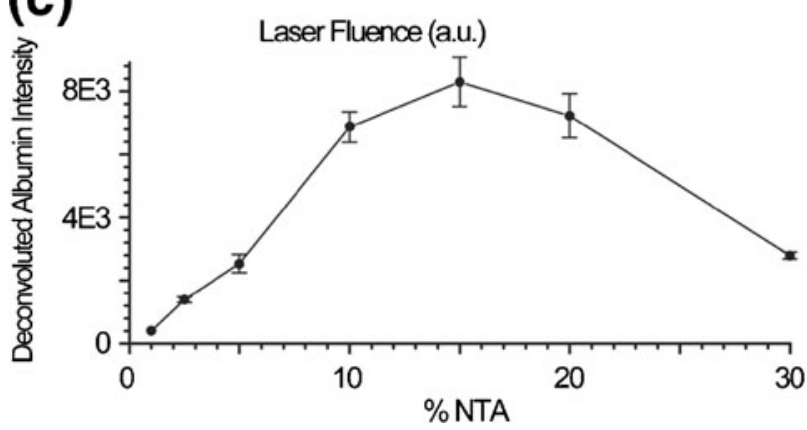

Figure 4. Characterization of MS-immunoassay parameters. (a) Albumin mass spectra as a function of laser fluence. (b) Deconvoluted albumin signal from methanol and DMSO VIC as a function of laser fluence. (c) Deconvoluted albumin signal intensity as a function of SAM functional groups (NTA ligands) surface density. All data were collected using $10^{5}$ dilute plasma experiments with co-added scans, successive shots should be performed at different areas of the surface.

Immunoassay Surface Density Previous investigations have shown that antibody fractional activity can be maximized by orienting the antigenic binding region toward the solution interface [49]. The monolayer used here was composed of alkanethiolates terminated with either NTA or EG3 ligands. The EG3 group served as a built-in blocking reagent to minimize non-specific interactions, while the NTA ligand provides an anchor for histidine-tagged protein $G$ which enables proper antibody orientation through the non-antigenic Fc domain [50]. Investigations have shown that controlled surface density of immobilized binding motifs enhanced surface interactions by minimizing steric exclusion [51]. A series of albumin MS-immunoassays were performed with biochips of varying NTA surface density $(1 \%-$ $30 \% \mathrm{~mol} / \mathrm{mol}$ ). A plot of the deconvoluted albumin signal (Figure $4 \mathrm{c}$ ) indicates maximum intensity at $\sim 15 \%$ NTA:EG3, consistent with previous work on enzymelinked immunosorbent assay (ELISA)s that suggest $<25 \%$ of randomly immobilized antibodies are surface active [49]. At $15 \%$ NTA density we estimate there is $\sim 1.16 \times 10^{-10} \mathrm{NTA} \mathrm{mol} / \mathrm{cm}^{2}$ [52]. With an estimated laser spot size of $\sim 100 \mu \mathrm{m}^{2}$ and a 2:1 albumin:NTA stoichiometry, we estimate that a maximum of 23 amol albumin were consumed per laser pulse highlighting the sensitivity of MALDI-TOF measurements of intact proteins from SAM immunoassays.

\section{Assay Performance}

We postulated that uniform distribution of analyte on SAMs facilitates reproducible SAMDI-TOF when supported by TLT/VIC matrix deposition. Table S4 summarizes intra- and inter-assay reproducibility observed for various MS-immunoassays performed. For example, deconvoluted signal intensity from 60 single-shot albumin immunoassay replicates (Figure S7) yielded $21 \%$ and $16 \%$ RSD for methanol VIC and DMSO VIC, respectively. For signal-averaged data (100 laser shots) the precision improved greatly. For example, for the different VIC solvent systems on $0.4 \times 0.5 \mathrm{~cm}$ biochips in Figure 2 the RSD averaged $\sim 3.7 \%$. Random sampling across $2.5 \times 2.5 \mathrm{~cm}$ albumin immunoassays yielded $<10 \%$ RSD for TLT/VIC, compared with $54 \%$ RSD for DD (Figure 5a versus 5b), with similar results observed for cystatin C (Figure S8). The TLT/ VIC application gives similar error for absolute measurements to that obtained by others for relative measurements with SAMDI [53]. Generally, the data show (Table S4) that TLT/VIC typically exhibit precisions of 3-9 \% RSD, with an average of $6.5 \%$, consistent with ELISA $(<10 \%$ RSD) [54], the gold standard for 

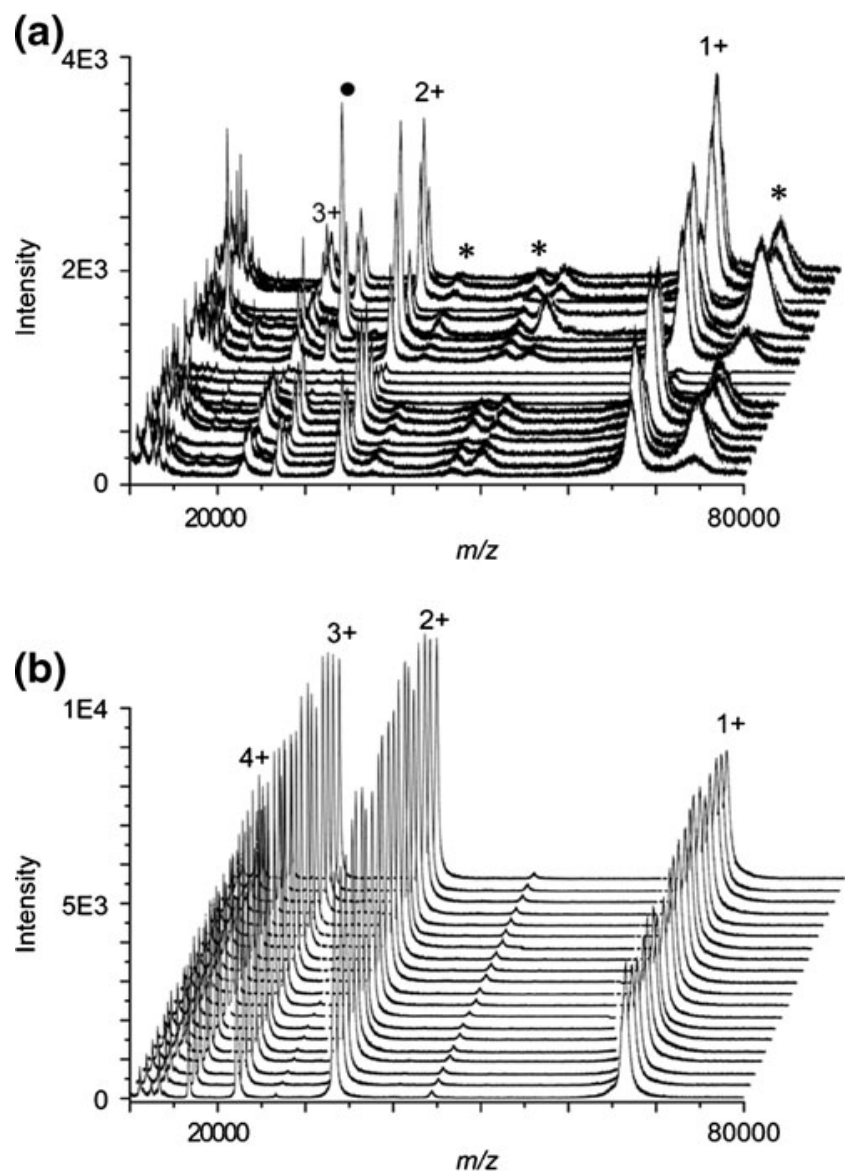

Figure 5. Reproducibility comparison of anti-albumin MSimmunoassays with DD and TLT/NICs. The 20 analogous mass spectra of anti-albumin incubated with $10^{5}$ dilute plasma obtained from (a) DD and (b) CHCA TLT with MEOH VIC. The ion peaks labeled with $(\bullet)$ and $\left({ }^{*}\right)$ are from protein $G$ and anti-albumin, respectively

diagnostic immunoassays, and monolayer-based immunoassays with SPR ( 4 \% RSD) [54].

Overall, we envision that our SAMDI immunoassays with TLT/VIC support reproducible protein analysis with the precision necessary for routine molecular diagnostic environments. Similar to other MALDI-immunoassay formats [7, 9-13], we anticipate diverse applications for this technology; including those that help to rationalize diverse sources of biological variation (e.g., alternative splicing, polymorphisms, and post-translational modifications). Future work will continue to develop this approach for quantitation of monolayer-bound proteins without the use of internal standards. Furthermore, we will evaluate the unique crystallization tendencies of other matrices and determine how matrix selection may improve detection of different molecular species in both the positive and negative ion modes.

\section{Conclusions}

We report the combination of antibody-coupled SAM biochips with a facile and rapid method for matrix deposition which enables sensitive and reproducible MS analysis of immobilized antigens. Robust label-free processing with MALDI-TOF is demonstrated using rationally designed substrates and methods that control matrix/analyte co-crystallization. The TLT method provides uniform matrix deposition over large surface areas without disturbing the immobilized analyte, while requiring only small $(50-100 \mu \mathrm{g})$ quantities of matrix. Coupling the TLT method to organic solvent VIC demonstrated improved MS signal intensities and intra- and inter-assay reproducibilities for proteins bound to SAM biochips compared with DD. The work also highlights how controlling solvent, matrix, and analyte interactions during crystallization impacts analyte charging and $\mathrm{S} / \mathrm{N}$. The data presented here suggest that TLT matrix deposition followed by organic solvent VIC may also be employed in diverse diagnostic SAMDI applications for sensitive, reproducible, and label-free protein molecular diagnostics.

\section{Acknowledgments}

The authors acknowledge support for this work by the University of Texas Southwestern Medical Center (UTSW), University of Texas at Dallas (UTD), and the John L. Roach Scholarship in Biomedical Research. The authors thank the Cleanroom Research Laboratory at UTD and the Molecular and Cellular Imaging Facility at UTSW for assistance with imaging studies.

\section{References}

1. Wingren, C., Borrebaeck, C.A.: Antibody-based microarrays. Methods Mol. Biol. 509, 57-84 (2009)

2. Mohammed, M.I., Desmulliez, M.P.: Lab-on-a-chip based immunosensor principles and technologies for the detection of cardiac biomarkers: a review. Lab Chip 11, 569-595 (2011)

3. Takamune, N., Hamada, H., Sugawara, H., Misumi, S., Shoji, S.: Development of an enzyme-linked immunosorbent assay for measurement of activity of myristoyl-coenzyme A: protein $N$-myristoyltransferase. Anal. Biochem. 309, 137-142 (2002)

4. Soderstrom, C.I., Spriggs, F.P., Song, W., Burrell, S.: Comparison of four distinct detection platforms using multiple ligand binding assay formats. J. Immunol. Methods 371, 106-113 (2011)

5. Scarano, S., Mascini, M., Turner, A.P., Minunni, M.: Surface plasmon resonance imaging for affinity-based biosensors. Biosens. Bioelectron. 25, 957-966 (2010)

6. Albertini, V., Bruno, A., Paterlini, A., Lista, S., Benussi, L., Cereda, C., Binetti, G., Ghidoni, R.: Optimization protocol for amyloid-beta peptides detection in human cerebrospinal fluid using SELDI TOF MS. Proteom. Clin. Appl. 4, 352-357 (2010)

7. Cornelius, R.M., Shankar, S.P., Brash, J.L., Babensee, J.E.: Immunoblot analysis of proteins associated with self-assembled monolayer surfaces of defined chemistries. J. Biomed. Mater. Res. A 98, 7-18 (2011)

8. Kausaite-Minkstimiene, A., Ramanaviciene, A., Kirlyte, J., Ramanavicius, A.: Comparative study of random and oriented antibody immobilization techniques on the binding capacity of immunosensor. Anal. Chem. 82, 6401-6408 (2010)

9. Nelson, R.W., Borges, C.R.: Mass spectrometric immunoassay revisited. J. Am. Soc. Mass Spectrom. 22, 960-968 (2011)

10. Baggerly, K.A., Morris, J.S., Coombes, K.R.: Reproducibility of SELDI-TOF protein patterns in serum: comparing datasets from different experiments. Bioinformatics 20, 777-785 (2004)

11. Reid, J.D., Holmes, D.T., Mason, D.R., Shah, B., Borchers, C.H.: Towards the development of an immunoMALDI (iMALDI) mass spectrometry assay for the diagnosis of hypertension. J. Am. Soc. Mass Spectrom. 21, 1680-1686 (2010) 
12. Evans-Nguyen, K.M., Tao, S.C., Zhu, H., Cotter, R.J.: Protein arrays on patterned porous gold substrates interrogated with mass spectrometry: Detection of peptides in plasma. Anal. Chem. 80, 1448-1458 (2008)

13. Patwa, T.H., Zhao, J., Anderson, M.A., Simeone, D.M., Lubman, D.M.: Screening of glycosylation patterns in serum using natural glycoprotein microarrays and multi-lectin fluorescence detection. Anal. Chem. 78, 6411-6421 (2006)

14. Szajli, E., Feher, T., Medzihradszky, K.F.: Investigating the quantitative nature of MALDI-TOF MS. Mol. Cell. Proteom. 7, 2410-2418 (2008)

15. Enebro, J., Karlsson, S.: Improved matrix-assisted laser desorption/ ionization time-of-flight mass spectrometry of carboxymethyl cellulose. Rapid Commun. Mass Spectrom. 20, 3693-3698 (2006)

16. Kim, Y., Hurst, G.B., Doktycz, M.J., Buchanan, M.V.: Improving spot homogeneity by using polymer substrates in matrix-assisted laser desorption/ionization mass spectrometry of oligonucleotides. Anal. Chem. 73, 2617-2624 (2001)

17. Axelsson, J., Hoberg, A., Waterson, C., Myatt, P., Shield, G.L., Varney, J., Haddleton, D.M., Derrick, P.J.: Improved reproducibility and increased signal intensity in MALDI as a result of electrospray sample preparation. Rapid Commun. Mass Spectrom. 11, 209-213 (1997)

18. Baluya, D.L., Garrett, T.J., Yost, R.A.: Automated MALDI matrix deposition method with inkjet printing for imaging mass spectrometry. Anal. Chem. 79, 6862-6867 (2007)

19. Bouschen, W., Schulz, O., Eikel, D., Spengler, B.: Matrix vapor deposition/recrystallization and dedicated spray preparation for highresolution scanning microprobe matrix-assisted laser desorption/ionization imaging mass spectrometry (SMALDI-MS) of tissue and single cells. Rapid Commun. Mass Spectrom. 24, 355-364 (2010)

20. Chen, Y., Allegood, J., Liu, Y., Wang, E., Cachon-Gonzalez, B., Cox, T.M., Merrill Jr., A.H., Sullards, M.C.: Imaging MALDI mass spectrometry using an oscillating capillary nebulizer matrix coating system and its application to analysis of lipids in brain from a mouse model of Tay-Sachs/ Sandhoff disease. Anal. Chem. 80, 2780-2788 (2008)

21. Chen, Y., Liu, Y., Allegood, J., Wang, E., Cachon-Gonzalez, B., Cox, T.M., Merrill Jr., A.H., Sullards, M.C.: Imaging MALDI mass spectrometry of sphingolipids using an oscillating capillary nebulizer matrix application system. Methods Mol. Biol. 656, 131-146 (2010)

22. Dekker, L.J., van Kampen, J.J., Reedijk, M.L., Burgers, P.C., Gruters, R.A., Osterhaus, A.D., Luider, T.M.: A mass spectrometry based imaging method developed for the intracellular detection of HIV protease inhibitors. Rapid Commun. Mass Spectrom. 23, 1183-1188 (2009)

23. Hankin, J.A., Barkley, R.M., Murphy, R.C.: Sublimation as a method of matrix application for mass spectrometric imaging. J. Am. Soc. Mass Spectrom. 18, 1646-1652 (2007)

24. Hensel, R.R., King, R.C., Owens, K.G.: Electrospray sample preparation for improved quantitation in matrix-assisted laser desorption/ ionization time-of-flight mass spectrometry. Rapid Commun. Mass Spectrom. 11, 1785-1793 (1997)

25. Jaskolla, T.W., Karas, M., Roth, U., Steinert, K., Menzel, C., Reihs, K. Comparison between vacuum sublimed matrices and conventional dried droplet preparation in MALDI-TOF mass spectrometry. J. Am. Soc. Mass Spectrom. 20, 1104-1114 (2009)

26. Jurchen, J.C., Rubakhin, S.S., Sweedler, J.V.: MALDI-MS imaging of features smaller than the size of the laser beam. J. Am. Soc. Mass Spectrom. 16, 1654-1659 (2005)

27. Wei, H., Nolkrantz, K., Powell, D.H., Woods, J.H., Ko, M.C., Kennedy, R.T.: Electrospray sample deposition for matrix-assisted laser desorption/ionization (MALDI) and atmospheric pressure MALDI mass spectrometry with attomole detection limits. Rapid Commun. Mass Spectrom. 18, 1193-1200 (2004)

28. Yang, J., Caprioli, R.M.: Matrix sublimation/recrystallization for imaging proteins by mass spectrometry at high spatial resolution. Anal. Chem. 83, 5728-5734 (2011)

29. D'Imperio, M., Della Corte, A., Facchiano, A., Di Michele, M., Ferrandina, G., Donati, M.B., Rotilio, D.: Standardized sample preparation phases for a quantitative measurement of plasma peptidome profiling by MALDI-TOF. J. Proteom. 73, 1355-1367 (2010)

30. Anderson, N.L., Razavi, M., Pearson, T.W., Kruppa, G., Paape, R., Suckau, D.: Precision of heavy-light peptide ratios measured by malditof mass spectrometry. J. Proteome Res. 11, 1868-1878 (2012)

31. Gurard-Levin, Z.A., Mrksich, M.: Combining self-assembled monolayers and mass spectrometry for applications in biochips. Annu. Rev. Anal. Chem. (Palo Alto, CA) 1, 767-800 (2008)
32. Mrksich, M.: Mass spectrometry of self-assembled monolayers: a new tool for molecular surface science. ACS Nano 2, 7-18 (2008)

33. Patrie, S.M., Mrksich, M.: Self-assembled monolayers for MALDI-TOF mass spectrometry for immunoassays of human protein antigens. Anal. Chem. 79, 5878-5887 (2007)

34. Lee, H.J., Nedelkov, D., Corn, R.M.: Surface plasmon resonance imaging measurements of antibody arrays for the multiplexed detection of low molecular weight protein biomarkers. Anal. Chem. 78, 6504-6510 (2006)

35. Sadeghi, M., Vertes, A.: Crystallite size dependence of volatilization in matrixassisted laser desorption ionization. Appl. Surf. Sci. 127, 226-234 (1998)

36. Qiao, H., Piyadasa, G., Spicer, V., Ens, W.: Analyte distributions in MALDI samples using MALDI imaging mass spectrometry. Int. J. Mass Spectrom. 281, 41-51 (2009)

37. Tarzi, O.I., Nonami, H., Erra-Balsells, R.: The effect of temperature on the stability of compounds used as UV-MALDI-MS matrix: 2,5dihydroxybenzoic acid, 2,4,6-trihydroxyacetophenone, $\longmapsto$-cyano-4hydroxycinnamic acid, 3,5-dimethoxy-4-hydroxycinnamic acid, norharmane, and harmane. J. Mass Spectrom. 44, 260-277 (2009)

38. Murphy, R.C., Hankin, J.A., Barkley, R.M., Zemski Berry, K.A.: MALDI imaging of lipids after matrix sublimation/deposition. Biochim. Biophys. Acta 1811, 970-975 (2011)

39. Poetsch, A., Schlusener, D., Florizone, C., Eltis, L., Menzel, C., Rogner, M., Steinert, K., Roth, U.: Improved identification of membrane proteins by MALDI-TOF MS/MS using vacuum sublimated matrix spots on an ultraphobic chip surface. J. Biomol. Tech. 19, 129-138 (2008)

40. Mascaro, D.J., Thompson, M.E., Smith, H.I., Bulovic, V.: Forming oriented organic crystals from amorphous thin films on patterned substrates via solvent-vapor annealing. Org. Electron. 6, 211-220 (2005)

41. Conboy, J.C., Olson, E.J.C., Adams, D.M., Kerimo, J., Zaban, A., Gregg, B.A., Barbara, P.F.: Impact of solvent vapor annealing on the morphology and photophysics of molecular semiconductor thin films. $J$. Phys. Chem. B 102, 4516-4525 (1998)

42. Beavis, R.C., Bridson, J.N.: Epitaxial protein inclusion in sinapic acid crystals. J. Phys. D Appl. Phys. 26, 442-447 (1993)

43. Cohen, S.L., Chait, B.T.: Influence of matrix solution conditions on the MALDI-MS analysis of peptides and proteins. Anal. Chem. 68, 31-37 (1996)

44. Jaskolla, T.W., Karas, M.: Compelling evidence for Lucky Survivor and gas phase protonation: the unified MALDI analyte protonation mechanism. J. Am. Soc. Mass Spectrom. 22, 976-988 (2011)

45. Knochenmuss, R., McCombie, G., Faderl, M.: Ion yields of thin MALDI samples: Dependence on matrix and metal substrate and implications for models. J. Phys. Chem. A 110, 12728-12733 (2006)

46. Iwatsu, F., Kobayashi, T., Uyeda, N.: Solvent effects on crystal-growth and transformation of zinc phthalocyanine. J. Phys. Chem. US 84, 3223-3230 (1980)

47. Granasy, L., Pusztai, T., Tegze, G., Warren, J.A., Douglas, J.F.: Growth and form of spherulites. Phys. Rev. E Stat. Nonlin. Soft Matter Phys $\mathbf{7 2}$, $011605(2005)$

48. Dreisewerd, K.: The desorption process in MALDI. Chem. Rev. 103, 395-425 (2003)

49. Peluso, P., Wilson, D.S., Do, D., Tran, H., Venkatasubbaiah, M., Quincy, D., Heidecker, B., Poindexter, K., Tolani, N., Phelan, M., Witte, K., Jung, L.S., Wagner, P., Nock, S.: Optimizing antibody immobilization strategies for the construction of protein microarrays. Anal. Biochem. 312, 113-124 (2003)

50. Mrksich, M., Whitesides, G.M.: Using self-assembled monolayers that present oligo(ethylene glycol) groups to control the interactions of proteins with surfaces. Poly (Ethylene Glycol) 680, 361-373 (1997)

51. Kwon, Y., Han, Z., Karatan, E., Mrksich, M., Kay, B.K.: Antibody arrays prepared by cutinase-mediated immobilization on self-assembled monolayers. Anal. Chem. 76, 5713-5720 (2004)

52. Nayak, S., Yeo, W.S., Mrksich, M.: Determination of kinetic parameters for interfacial enzymatic reactions on self-assembled monolayers. Langmuir 23, 5578-5583 (2007)

53. Gurard-Levin, Z.A., Scholle, M.D., Eisenberg, A.H., Mrksich, M.: High-throughput screening of small molecule libraries using SAMDI mass spectrometry. ACS Comb. Sci. 13, 347-350 (2011)

54. Sakai, S., Matsuda, R., Adachi, R., Akiyama, H., Maitani, T., Ohno, Y., Oka, M., Abe, A., Seiki, K., Oda, H., Shiomi, K., Urisu, A.: Interlaboratory evaluation of two enzyme-linked immunosorbent assay kits for the determination of crustacean protein in processed foods. $J$. AOAC Int. 91, 123-129 (2008) 